\title{
Cooperación en modelos de Cournot con información incompleta
}

\section{ROSA ARRANZ SOMBRÍA}

Departamento de Economía Aplicada, UNIVERSIDAD DE VALLADOLID, ESPAÑA. Email: rosa@eco.uva.es

\section{RESUMEN}

Se estudia el oligopolio de Cournot donde el proceso de ajuste de la producción se realiza considerando que las empresas carecen de información completa sobre el comportamiento de sus competidoras. En este contexto, suponiendo la intención cooperativa como factor fundamental, se alcanzan equilibrios cooperativos empleando sistemas dinámicos discretos que tienen en cuenta la interacción repetida de las empresas. El trabajo muestra que la cooperación es posible sin necesidad de acuerdo previo.

Palabras clave: Oligopolio, información incompleta, cooperación.

\section{Cooperation in Cournot's Models with Incomplete Information}

\begin{abstract}
Cournot oligopoly is studied under the circumstances that firms do not have complete information about their competitors' behaviour. In this context, assuming that cooperative intention is the main aspect, we can reach cooperative equilibriums by employing discrete dynamical systems that take into account repeated interactions between firms. This paper shows that cooperation is possible without a previous agreement.
\end{abstract}

Keywords: Oligopoly, incomplete information, cooperation.

Clasificación JEL: C72, D43, C30

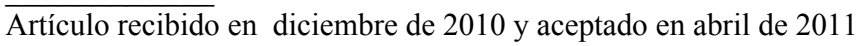

Artículo disponible en versión electrónica en la página www.revista-eea.net, ref. ə-29112 


\section{INTRODUCCIÓN}

La teoría de juegos ha sido ampliamente utilizada en el estudio de los oligopolios gracias a su capacidad para considerar situaciones de interacción estratégica entre las empresas. El oligopolio es en esencia competitivo y la solución básica del juego en su versión estática es el equilibrio de Nash. Éste se basa en condiciones de racionalidad bastante exigentes, que incluyen supuestos restrictivos sobre la información disponible y sobre la capacidad de las empresas para obtener estimaciones correctas acerca del comportamiento de las otras empresas, además de implicar la solución de problemas de optimización, que involucran un elevado coste computacional. La interacción entre las empresas en un mercado tiene un comportamiento fundamentalmente dinámico. El proceso de ajuste para alcanzar el equilibrio de Nash y el estudio de su estabilidad se ha llevado a cabo en numerosos trabajos y desde diferentes perspectivas (Agiza y Elsadany (2003), Naimzada y Sbragia (2006).

Una de las cuestiones más significativas del equilibrio de Nash en el oligopolio de Cournot es que refleja la racionalidad individual pero no la colectiva (la solución de Nash no es un óptimo de Pareto). El juego del dilema del prisionero muestra claramente la contradicción entre los intereses individuales y los colectivos y que las elecciones basadas en la maximización de los intereses individuales no conducen necesariamente a un óptimo social. Una de las cuestiones principales que plantea el dilema del prisionero es si un comportamiento cooperativo puede surgir entre jugadores egoístas y racionales sin un acuerdo previo cuando se juega varias veces. La respuesta es negativa si el juego se repite un número finito de etapas, pero no lo es cuando el juego es infinito, donde los llamados teoremas folk muestran que la cooperación es posible estableciendo un sistema basado en recompensas y castigos en cada etapa. Axelrod (1984) probó que la mejor estrategia para la consecución de la cooperación es la tit-for-tat, que consiste en hacer lo que el oponente hizo en el movimiento anterior.

Las ideas anteriores se pueden extender fácilmente al contexto de un oligopolio donde se puede considerar que unas pocas empresas interactúan de forma repetida. El desconocimiento del final del proceso permite considerarlo como un juego con horizonte infinito. Sin embargo, las variables de control (cantidades o precios), a diferencia del dilema del prisionero, tienen un amplio rango de variación, lo que no permite una definición clara de lo que es un comportamiento cooperativo o no cooperativo ni tampoco lo que es recompensa o castigo.

Cafagna y Coccorese (2005) construyen en un duopolio lineal de Cournot, una estrategia de ajuste en el output que conduce a un equilibrio cooperativo. La estrategia que emplea está basada en el conocimiento por parte de ambas empresas de los outputs y beneficios de la otra, es decir, las estrategias se 
definen en un marco de información completa. Sin embargo, es habitual que empresas competidoras, o incluso si se ha alcanzado cierta cooperación, desconozcan los productos y beneficios de las otras. Por este motivo, el supuesto de información incompleta es más realista y va a ser el escenario utilizado en este trabajo. La cuestión es si vamos a encontrar un proceso de ajuste del output que permita a las empresas alcanzar un equilibrio cooperativo sin un acuerdo previo.

Ding y Shi (2009) estudian el mismo modelo que Cafagna y Coccorese (2005) en un contexto de información incompleta considerando dos tipos de estrategias. En el primero de los casos establece un proceso de ajuste del output basado en la diferencia entre el beneficio cooperativo y el generado por la empresa encontrando problemas con la estabilidad. Esta se consigue en el modelo cuando se incorpora la intención cooperativa a la estrategia de las empresas (Falk et al (2008)). Desde nuestro punto de vista, el ingrediente fundamental para conseguir la cooperación, y con ello un incremento en los beneficios, es pensar que ésta es el objetivo. Desde luego, no se trata de ser altruista y cooperar en cualquier circunstancia. Las empresas saben que cooperando pueden mejorar sus beneficios, y la idea básica es que así lo harán, salvo que con ello resulten perjudicadas. Mientras que las empresas obtengan rendimientos extraordinarios, continuarán cooperando.

Con este propósito, basándonos en las ideas utilizadas por Cafagna y Coccorese (2005) y por Ding y Shi (2009), vamos a definir las estrategias de cada empresa teniendo en cuenta su propia información, es decir, en un contexto de información incompleta. Vamos a estudiar si las empresas pueden conseguir mayores beneficios que los que se pueden alcanzar en el equilibrio de Cournot, que se van a considerar mínimos, ajustando sus producciones hacia lo que hemos denominado producto cooperativo. A diferencia de Cafagna y Coccorese (2005), nuestra referencia va a ser el beneficio no cooperativo que es el que proporciona el equilibrio de Nash y que se podría conseguir en ausencia de cooperación. Este se incorpora a la dinámica del modelo como criterio de decisión, no como parte explícita de las ecuaciones que determinan el comportamiento de las empresas.

El trabajo está organizado de la siguiente forma. En la sección 2 exponemos la versión general del modelo que vamos a estudiar. La sección 3 describe las estrategias que van a seguir los jugadores para alcanzar soluciones cooperativas. Las simulaciones numéricas que evidencian la consecución de los objetivos en distintos modelos se pueden ver en el apartado 4. Por último, en la sección 5 se recogen las conclusiones. 


\section{MODELO}

Vamos a considerar un oligopolio en el que $n$ empresas producen un bien homogéneo y donde las decisiones de producción se toman en periodos de tiempo discretos, $t=0,1,2 \ldots$ Denotamos por $q_{i, t}$, la cantidad de dicho bien producida por la empresa $i$ en el periodo $t$. El precio del output depende de la producción total de la industria de acuerdo a una función de demanda inversa $p_{t}=f\left(q_{t}\right)$ donde $q_{t}=\sum_{i=1}^{n} q_{i, t}$ es el output total del mercado en $t$. Si el coste de producción para la empresa $i$ viene dado por $C_{i}\left(q_{i, t}\right)$, entonces su beneficio para el periodo $t$ es

$$
\pi_{i, t}=p_{t} q_{i, t}-C_{i}\left(q_{i, t}\right) .
$$

Bajo ciertas condiciones de regularidad para las funciones de demanda inversa y de costes, se puede asegurar que el oligopolio de Cournot estático tiene un único equilibrio de Nash con cantidades estrictamente positivas (Pérez et al 2004) que denotamos por $q_{n c}^{i}$. El beneficio que obtiene la empresa $i$ en el equilibrio es $\pi_{n c}^{i}$. El subíndice $n c$ hace referencia a que se pueden obtener sin cooperación.

En nuestro modelo necesitamos determinar cómo van a decidir las empresas las cantidades a producir en el periodo $t+1$ como respuesta a los beneficios alcanzados en el periodo $t$. Como indicamos en la introducción, asumimos que cada empresa desconoce las cantidades producidas por las otras $\mathrm{y}$, por lo tanto, desconoce también sus beneficios. Cuando una empresa tome sus decisiones de producción para el periodo $t+1$, va a utilizar como referencia sus beneficios del periodo $t, \pi_{i, t} \mathrm{y}$ la cantidad producida $q_{i, t}$, que son los datos que conoce. Los estrategias están basadas en que las empresas van a comparar sus beneficios $\pi_{i, t}$ con los no cooperativos $\pi_{n c}^{i}$. El objetivo es alcanzar un beneficio mayor que vamos a llamar beneficio cooperativo y que denotamos por $\pi_{c}^{i}$.

Cafagna y Coccorese (2005) y Ding y Shi (2009) estudian el caso de un duopolio lineal donde las empresas tienen el mismo tamaño y proponen como solución cooperativa el reparto de los beneficios del monopolio. Nosotros hemos adoptado el mismo criterio en el apartado 4.1. Sin embargo, esta idea no es extrapolable a otros contextos, como el caso en el que los costes son cuadráticos o en el que las empresas tienen diferentes costes de producción. El beneficio cooperativo va a depender de las características concretas de cada modelo aunque su determinación tiene un argumento común.

La interacción repetida de las empresas a lo largo del tiempo produce una sucesión de niveles de producción $\left\{q_{i, 1}, q_{i, 2}, \ldots, q_{i, t}, \ldots\right\}$ que generan una sucesión de beneficios $\left\{\pi_{i, 1}, \pi_{i, 2}, \ldots, \pi_{i, t}, \ldots\right\}$. Supondremos que en función de ellos, la 
empresa $i$ elige el nuevo nivel de producción, $q_{i, t+1}=g_{i}\left(q_{i, t}, \pi_{i, t}\right)$. La forma en la que se elige se explica en el apartado siguiente.

\section{ESTRATEGIA COOPERATIVA}

En este apartado vamos a establecer cómo determinan las empresas las cantidades que van a producir en el periodo $t+1$ una vez que conocen los beneficios que se han obtenido en el periodo $t$.

Nuestra formulación toma como referencia el modelo propuesto por Smale (1980) para la solución del juego del dilema del prisionero en su versión repetida. En él, las estrategias se definen tomando como referencia los beneficios que se obtienen. En ese juego sólo se puede elegir entre dos opciones, confesar o no confesar, que serían asimilables a las cantidades cooperativas y no cooperativas. Este hecho introduce una importante diferencia con el contexto productivo del oligopolio donde hay infinitas posibilidades para la producción. Además es razonable pensar que las empresas no pueden llevar a cabo modificaciones drásticas en su producción por lo que parece sensato plantearse un cambio gradual tanto hacia el output cooperativo como al no cooperativo.

Cafagna y Coccorese (2005) utilizan las ideas de Smale (1980) en un modelo de duopolio con información completa restringiendo el conjunto de posibles outputs a cantidades superiores al reparto del monopolio e inferiores a lo que denomina el output del desvío unilateral. En nuestro caso no vamos a introducir ningún tipo de restricción en la producción, aunque se consideran aquellas producciones que desde un punto de vista lógico tienen sentido; es decir, por una parte las que no generan precios negativos y por otra las que implican beneficios positivos. Por otro lado, no es razonable que una empresa produzca un output mayor cuando tiene que repartirse el mercado con otras, que cuando tiene el monopolio de éste. La producción del monopolio va a ser considerada como el techo productivo.

Para definir la estrategia de la empresa, vamos a utilizar un sencillo modelo de ajuste donde se van a tomar como referencia de beneficios mínimos aquellos que pueden obtener las empresas sin llegar a un acuerdo, es decir, tomamos como suelo para el beneficio el proporcionado por el equilibrio de Nash y que hemos denotado por $\pi_{n c}^{i}$. Desde la perspectiva de la empresa $i$, un beneficio mayor a éste implica una cooperación no acordada con las otras empresas por lo que, como recompensa, se propone reducir su producción con la intención de alcanzar el beneficio considerado como cooperativo y denotado por $\pi_{c}^{i}$. Si los beneficios son menores que $\pi_{n c}^{i}$, ello es indicativo de que los oponentes no están colaborando y la empresa se plantea, como castigo, moverse hacia la cantidad no cooperativa. 
En nuestro modelo, las empresas planifican su producción para el periodo $t+1$ teniendo en cuenta la producción en $t$ y la diferencia entre ésta y el equilibrio que desean alcanzar. Las ecuaciones dinámicas que definen el modelo son

$$
\begin{aligned}
& q_{i, t+1}=q_{i, t}+\alpha_{i}\left(q_{c}^{i}-q_{i, t}\right) \text { si } \pi_{i, t} \geq \pi_{n c}^{i}-\varepsilon_{i}, i=1,2, \ldots, n, 0<\alpha_{i}<1 \text { y } \varepsilon_{i}>0, \\
& q_{i, t+1}=q_{i, t}+\beta_{i}\left(q_{n c}^{i}-q_{i, t}\right) \text { si } \pi_{i, t}<\pi_{n c}^{i}-\varepsilon_{i}, i=1,2, \ldots, n, 0<\beta_{i}<1 \text { y } \varepsilon_{i}>0 .
\end{aligned}
$$

El tamaño de los parámetros de ajuste $\alpha_{i}$ y $\beta_{i}$ pretende medir la tendencia cooperativa o no de las empresas y $\varepsilon_{i}$ cierta tolerancia de las empresas al comparar con el beneficio no cooperativo. Observemos que se trata de ecuaciones en diferencias de primer orden cuyos equilibrios son precisamente $q_{c}^{i}$ y $q_{n c}^{i}$ que son asintóticamente estables para el rango de los valores de los parámetros de ajuste. El hecho de que cada empresa maneje simultáneamente dos dinámicas distintas podría desestabilizar el modelo, sin embargo en ninguna de las simulaciones realizadas se ha dado ese caso. En algunos ejemplos la dinámica del modelo tiene dos fases, en la primera las cantidades tienden a la solución no cooperativa y en la segunda, el output disminuye convergiendo a la cantidad cooperativa. En otros ejemplos el modelo converge directamente al producto cooperativo. Esta diferencia cualitativa depende de las condiciones iniciales de la simulación.

\section{MODELOS $Y$ CANTIDADES COOPERATIVAS. SIMULACIONES}

En esta sección vamos a exponer los distintos modelos estudiados, los métodos seguidos para obtener los outputs cooperativos y los resultados de las simulaciones para valores concretos de los parámetros.

\subsection{Oligopolio lineal con costes iguales}

Asumimos que la función de demanda inversa es lineal y que los costes de producción son lineales e iguales para todas las empresas, es decir,

$$
p_{t}=a-b q_{t} \text { y } C_{i}\left(q_{i, t}\right)=c q_{i, t}
$$

donde $a, b>0$ y $a>c$. En esta situación el equilibrio del modelo es el de NashCournot donde el output de cada empresa y los correspondientes beneficios son

$$
q_{n c}^{i}=q_{n c}=\frac{a-c}{(n+1) b} \quad \text { y } \quad \pi_{n c}^{i}=\pi_{n c}=\frac{(a-c)^{2}}{(n+1)^{2} b} .
$$


Como en otros trabajos, la situación cooperativa que vamos a considerar va a corresponder al caso en el que las empresas actúan como un monopolio. Bajo esta hipótesis y asumiendo que siendo iguales las empresas se repartirán en igual manera el aumento en los beneficios, las cantidades y beneficios cooperativos son

$$
q_{c}^{i}=q_{c}=\frac{a-c}{2 n b} \quad \mathrm{y} \quad \pi_{c}^{i}=\pi_{c}=\frac{(a-c)^{2}}{4 n b} .
$$

\subsubsection{Duopolio}

Comenzamos el análisis para el caso del duopolio. En la figura 1 se representan los valores cooperativos y no cooperativos para $a=8, b=1$ y $c=2$. Hemos elegido estos valores porque han sido utilizados en otros estudios Cafagna y Coccorese (2005) y Ding y Shi (2009)- y son los que vamos a emplear en las simulaciones numéricas. Cualquier distribución de cantidades que esté comprendida entre las curvas $C_{1}$ y $C_{2}$ (de trazo continuo), genera mayores beneficios para ambas empresas que la situación de equilibrio, es por ello que cualquiera de estas situaciones es más deseable que la de Cournot. De todas ellas, la que es óptima en cuanto a beneficios y además supone una distribución equitativa del mercado es el reparto del monopolio (punto de corte de las curvas de trazo discontinuo).

Figura 1.

Cantidades que mejoran los beneficios del equilibrio de Nash.

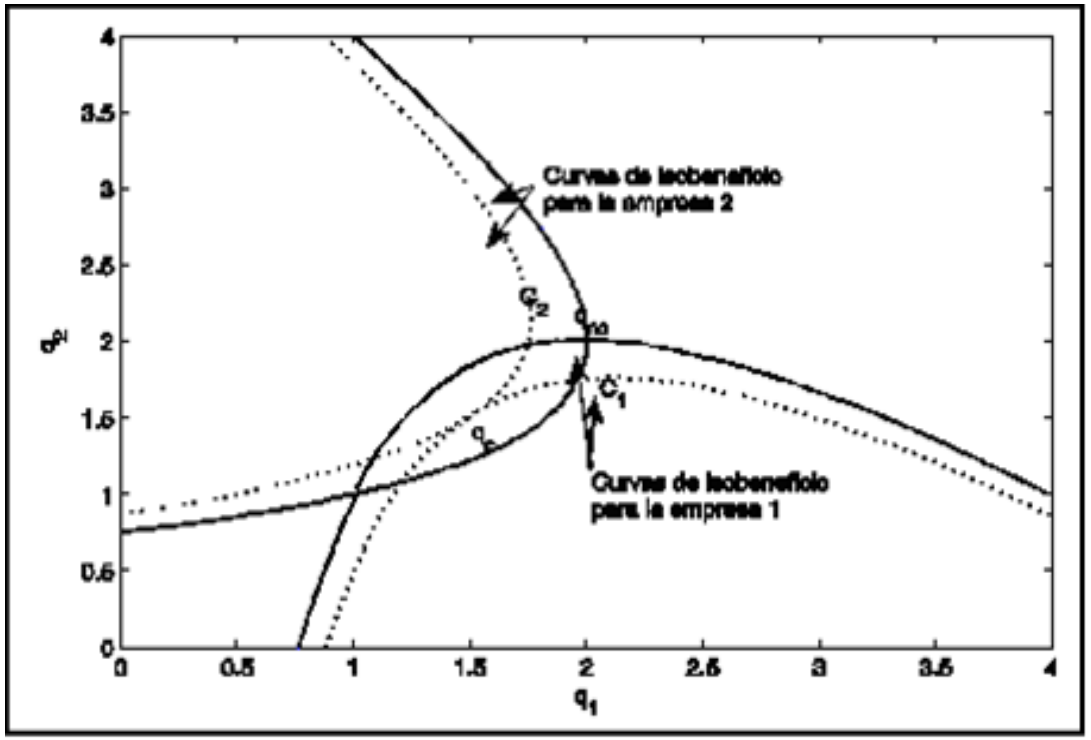


En este ejemplo, las cantidades son $q_{c}=1.5 \mathrm{y} q_{n c}=2 \mathrm{y}$ los beneficios $\pi_{c}=$ $4.5 \mathrm{y} \pi_{\mathrm{nc}}=4$. En primer lugar hemos trabajado considerando la igualdad de los parámetros de ajuste, $\alpha_{i}=\beta_{i}=0.01$, y para la tolerancia $\varepsilon_{i}=0.0001$ cuyo valor permanece constante en todas las simulaciones realizadas en este trabajo. En la figura 2 se recoge la evolución tanto de las cantidades producidas como de los beneficios generados.

Para analizar la convergencia de las estrategias propuestas al equilibrio cooperativo, hemos llevado a cabo diferentes simulaciones en función de las distintas características de las condiciones iniciales. En cada simulación se han realizado 2000 iteraciones aunque la convergencia se obtiene, en general, en las primeras etapas.

Hemos realizado simulaciones con diferentes condiciones iniciales intentando ver si la evolución de las estrategias depende de la relación de éstas con las cantidades cooperativas y no cooperativas. Hemos considerado seis situaciones que se pueden considerar como representativas. En la primera de ellas, partimos del equilibrio no cooperativo $\left(q_{1}=q_{2}=2\right)$, y podemos observar una convergencia rápida a las cantidades cooperativas donde las curvas para las dos empresas se solapan perfectamente. En la segunda hemos estudiado el caso en que las dos cantidades iniciales son inferiores al nivel cooperativo $\left(q_{1}=0.3\right.$, $\left.q_{2}=0.6\right)$ y además generan para ambas empresas beneficios menores que el equilibrio de Cournot. El comportamiento del modelo es bueno produciéndose un aumento sostenido del output hasta llegar a los niveles cooperativos. En el tercero analizamos cantidades inferiores a las cooperativas pero con beneficios superiores a los del equilibrio de Cournot $\left(q_{1}=1.3, q_{2}=1.1\right)$. En el cuarto, $q_{1}=1.3$, $q_{2}=1.7$, la primera es inferior a $q_{c}$ y la segunda está entre $q_{c}$ y $q_{n c}$. Para $q_{1}=0.3$, $q_{2}=2.7$ la primera empresa incrementa su producción y la segunda la reduce llegando fácilmente al equilibrio y por último en la situación sexta se estudian situaciones de beneficios inferiores al equilibrio de Cournot donde ambas empresas están produciendo por encima de los niveles de equilibrio. Podríamos decir que el ajuste tiene dos fases, en la primera las empresas están en una fase no cooperativa moviéndose hacia el equilibrio de Cournot y posteriormente se inicia la cooperación alcanzándose el beneficio cooperativo. 
Figura 2.

Dinámicas de cantidades y beneficios. Las condiciones iniciales de cada simulación aparecen en el título de cada una de las gráficas.

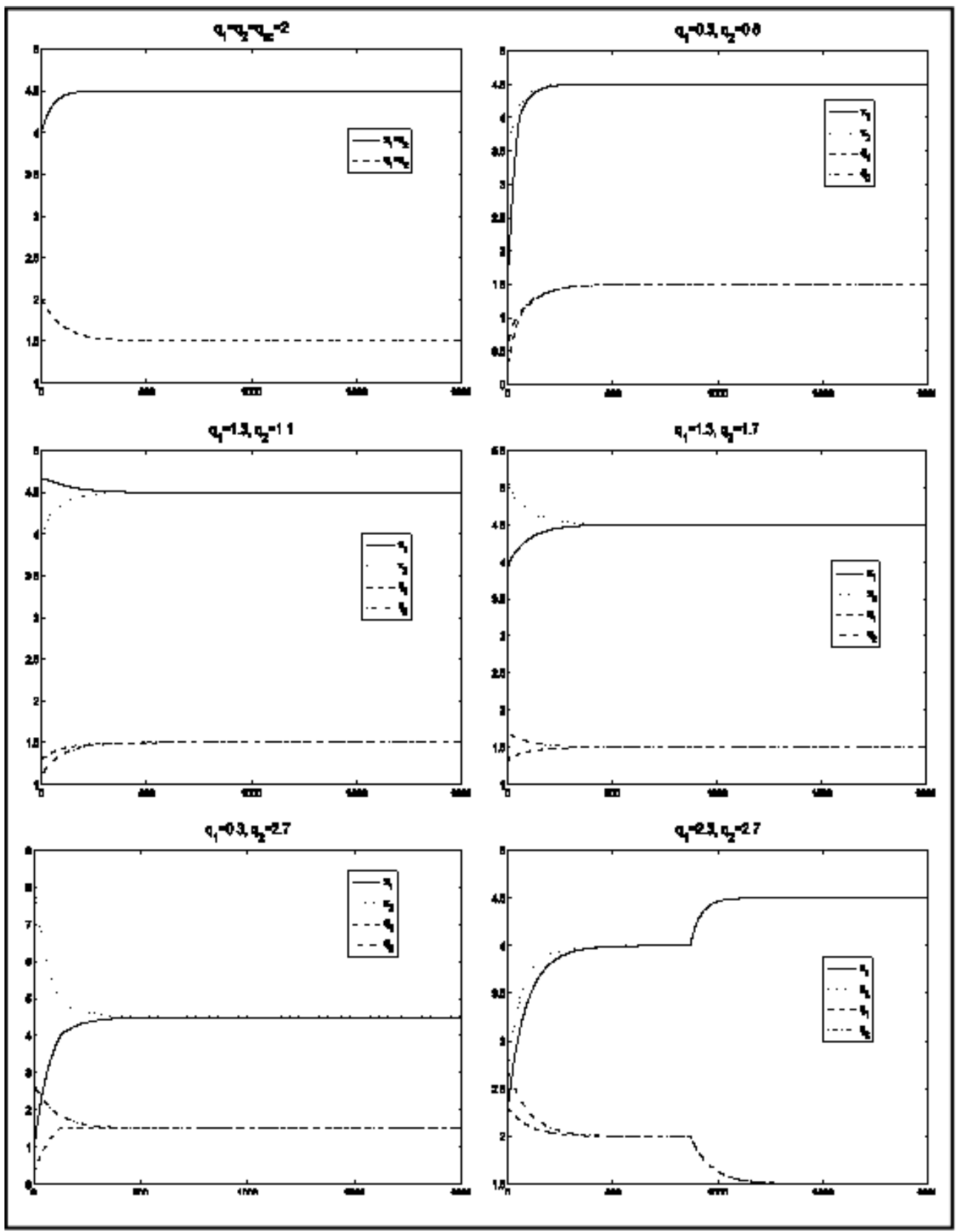


Después de todas las simulaciones realizadas podemos concluir que si ambas empresas emplean estas estrategias, se produce una convergencia hacia el producto cooperativo generando un aumento en los beneficios para ambas empresas.

Hemos realizado simulaciones con las mismas condiciones iniciales y con los valores de ajuste de los parámetros utilizados por Cafagna y Coccorese (2005), $\alpha_{i}=0.01$ y $\beta_{i}=0.005$, no encontrando diferencias significativas con las ya mostradas. En general el comportamiento es similar detectándose en algunos casos un ligero retraso en la convergencia hacia el equilibrio cooperativo. Esto es debido a que la actitud cooperativa medida por $\beta_{i}$ es menor que la no cooperativa medida por $\alpha_{i}$. Lo más destacable es que en la situación sexta, el salto hacia la situación cooperativa se produce alrededor de la iteración 1500 mientras que en la situación anterior es en torno a la 900. Por último estudiamos los parámetros $\alpha_{i}=0.01$ y $\beta_{i}=0.02$. En este caso el comportamiento cualitativo es similar pero parece que se adelanta la convergencia. En concreto en la situación sexta el paso a la situación cooperativa se sitúa en la iteración 450 .

\subsubsection{Más de dos empresas}

Dado que la estrategia que emplea una empresa depende de su propio producto y beneficio, pensamos que no hay ninguna razón para que el procedimiento no funcione cuando en el mercado operan más de dos empresas. Considerándolo como un ejercicio de comprobación, hemos realizado simulaciones siguiendo las mismas pautas que en el apartado 4.1.1. y con diferentes valores de los parámetros, cuando $\alpha_{i}=\beta_{i}=0.01$. En la figura 3 se representan como ejemplo dos de ellas. Todas las simulaciones confirman nuestras sospechas encontrando convergencia hacia la solución cooperativa. Estos resultados nos animan a concluir que en un oligopolio lineal con costes iguales, no es necesario firmar un acuerdo previo para desarrollar un comportamiento cooperativo y obtener incrementos en los beneficios. 
Figura 3.

Evolución de beneficios y cantidades. En el título de la gráfica aparecen las cantidades iniciales y en la parte inferior los valores de los parámetros y el número de empresas. Las líneas continuas representan los beneficios y las de puntos las cantidades.

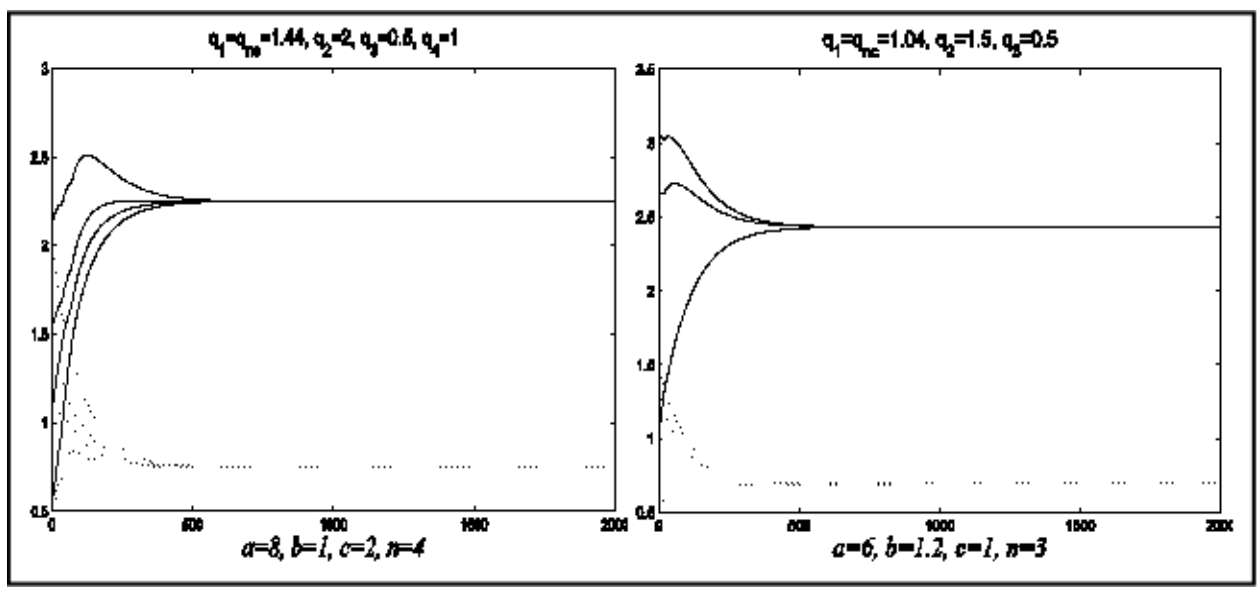

\subsection{Duopolio no lineal con costes iguales}

Debido a los buenos resultados obtenidos en el caso lineal con costes iguales, nos planteamos ampliar el estudio a otros modelos. Como ejemplo no lineal analizamos el duopolio estudiado por Naimzada y Sbragia (2006) que proponen la función de demanda inversa

$$
p_{t}=a-b \sqrt{q_{t}}
$$

y funciones de costes cuadráticos que se encuentran frecuentemente en la literatura, Negishi (1961) y Szidarovszky y Okuguchi (1998),

$$
C_{i}\left(q_{i, t}\right)=c_{i 0}+c_{i 1} q_{i, t}+c_{i 2} q_{i, t}^{2} .
$$

En esta situación la función de beneficios para la empresa $i$ toma la forma

$$
\pi_{i, t}=q_{i, t}\left(a-b \sqrt{q_{1, t}+q_{2, t}}\right)-\left(c_{i 0}+c_{i 1} q_{i, t}+c_{i 2} q_{i, t}^{2}\right) .
$$

Consideramos el modelo simétrico donde $\mathrm{c}_{10}=\mathrm{c}_{20}=0, \mathrm{c}_{11}=\mathrm{c}_{21}=\mathrm{c}_{1}$ y $\mathrm{c}_{12}=\mathrm{c}_{22}=\mathrm{c}_{2}$, y $a>c_{1}$. El único equilibrio de Nash simétrico del modelo (ver Bischi et al, 2007) satisfaciendo la condición $q \leq\left(a-c_{1}\right) /\left(2 c_{2}\right)$ viene dado por la expresión

$$
q^{*}=\frac{a-c_{1}}{2 c_{2}}+\frac{\left.5 b\left(5 b-\sqrt{25 b^{2}+64\left(a-c_{1}\right.}\right) c_{2}\right)}{64 c_{2}^{2}} .
$$


Siguiendo con la idea desarrollada en el apartado 4.1, esta va a ser la cantidad no cooperativa, $q^{*}=q_{n c}$. Análogamente al apartado anterior, nos planteamos como cantidad cooperativa el reparto del monopolio que se obtiene resolviendo el correspondiente problema de maximización donde la función objetivo es

$$
\pi(q)=q(a-b \sqrt{q})-\left(c_{1} q+c_{2} q^{2}\right)
$$

y cuya solución verificando la restricción es

$$
q_{\text {mon }}=\frac{\left(\sqrt{9 b^{2}+32 a c_{2}-32 c_{1} c_{2}}-3 b\right)^{2}}{64 c_{2}^{2}} \text { y con ello } q_{c}=\frac{q_{\text {mon }}}{2} .
$$

La no linealidad del modelo nos lleva a que esta elección no sea adecuada existiendo otras producciones que son mejores para ambas empresas. Por ejemplo, si $b=1$ y $c_{1}=1 \quad c_{2}=0.3$, el comportamiento cualitativo es diferente dependiendo del valor de $a$. En la tabla 1 podemos ver que para $a=8$, el reparto del monopolio puede llevar a situaciones peores que el equilibrio de Cournot.

Tabla 1

Cantidades y beneficios cooperativos y del monopolio

\begin{tabular}{|c|c|c|c|c|}
\hline & $q_{n c}$ & $q_{c}$ & $\pi_{n c}$ & $\pi_{c}$ \\
\hline$a=8$ & 5.0474 & 2.8496 & 11.6522 & 10.7078 \\
\hline$a=3$ & 0.7618 & 0.4634 & 0.4092 & 0.4162 \\
\hline
\end{tabular}

En la figura 4 se representa la situación de ambos casos. Estos dos ejemplos muestran claramente que no es adecuado elegir como solución cooperativa el reparto del monopolio. No obstante, existen distribuciones que mejoran la situación del equilibrio de Cournot. 
Figura 4.

Representación de la situación de los beneficios del monopolio para diferentes valores del parámetro.

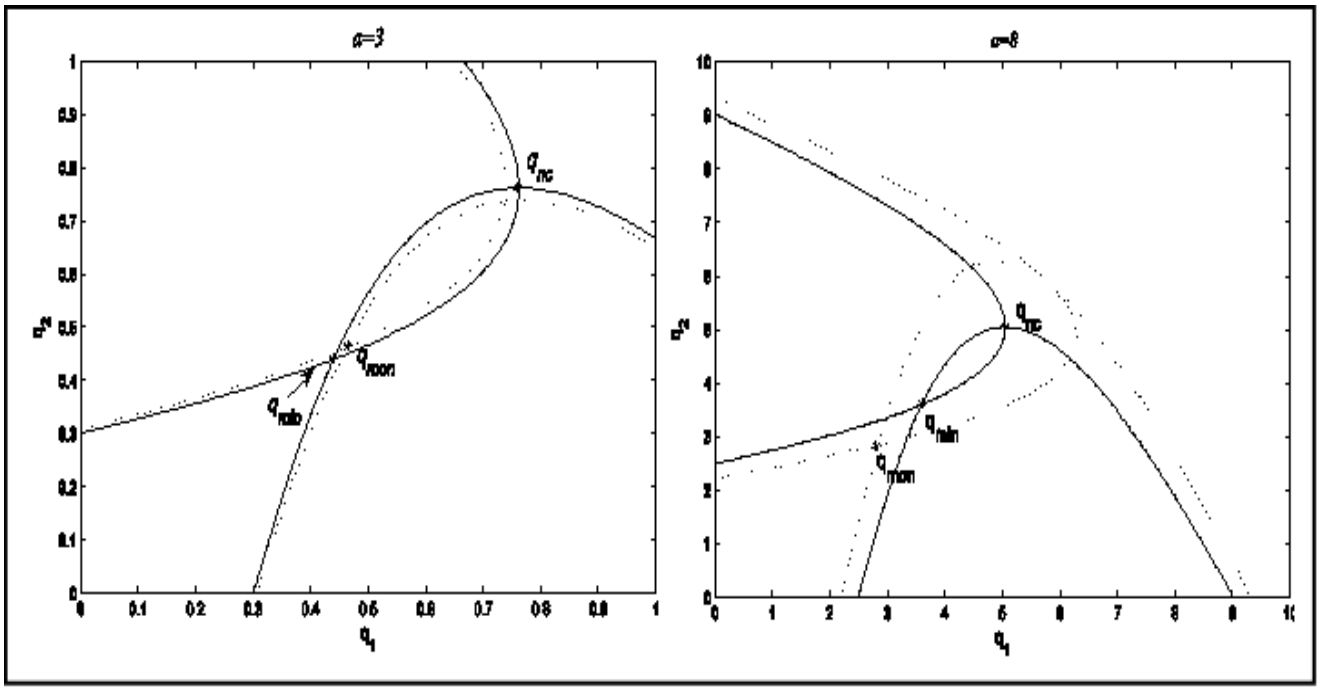

La idea fundamental no es elegir el producto del monopolio sino plantear una reducción en las producciones que nos lleve a situaciones de mayor beneficio que las generadas por el equilibrio de Cournot. Para determinar el producto cooperativo analizamos la evolución de los beneficios de cada empresa tomando como referencia cantidades que son inferiores al producto de Cournot y superiores a unas cantidades mínimas ${ }^{1}$ que hemos denotado por $q_{\min }=\left(q_{\min }^{1}, q_{\min }^{2}\right)$ y que vienen determinadas por el otro punto de corte de las curvas de isobeneficio que pasan por el mencionado equilibrio (ver figura 4). Dado que las empresas tienen el mismo tamaño, asumimos que las cantidades producidas por ambas son iguales y que están comprendidas entre $q_{\min } \mathrm{y} q_{n c}$. Los beneficios crecen hasta llegar a un máximo para empezar después a decrecer. La cantidad que genera el beneficio máximo es la que se toma como $q_{c}$; es decir, es el máximo de

$$
\pi(q)=q(a-b \sqrt{2 q})-\left(c_{1} q+c_{2} q^{2}\right), \text { donde } q_{\min } \leq q \leq q_{n c} .
$$

Por lo tanto,

\footnotetext{
${ }^{1}$ Cantidades inferiores a $q_{\min }$ implican beneficios inferiores a los del equilibrio de Cournot.
} 


$$
q_{c}=\frac{\left(\sqrt{9 b^{2}+16 a c_{2}-16 c_{1} c_{2}}-3 b\right)^{2}}{32 c_{2}^{2}} .
$$

Aplicando este procedimiento para el duopolio lineal estudiado en 4.1.1 se generan precisamente las cantidades que se obtienen en el reparto del monopolio. La determinación de las cantidades mínimas de forma explícita no es sencilla debido a la no linealidad del modelo. Por ello, en los ejemplos simulados hemos utilizado software específico para la resolución numérica de sistemas de ecuaciones no lineales. Para $a=8$, se tiene $q_{\min }=3.600$ y $q_{c}=$ 4.3190 , mientras que para $a=3$, los valores son $q_{\min }=0.4400$ y $q_{c}=0.5984$.

En la figura 5 hemos representado algunas de las simulaciones realizadas para $a=3$ y en la figura 6 los resultados para $a=8$. En ambos casos se ha considerado la igualdad de los parámetros de ajuste $\alpha_{i}=\beta_{i}=0.01$. Como se puede ver, una vez que están determinadas las cantidades cooperativas la convergencia hacia ellas es similar al modelo lineal.

Figura 5.

Convergencia de beneficios y cantidades hacia los valores cooperativos para $a=3$. Las condiciones iniciales de cada simulación aparecen en el título de cada gráfica.

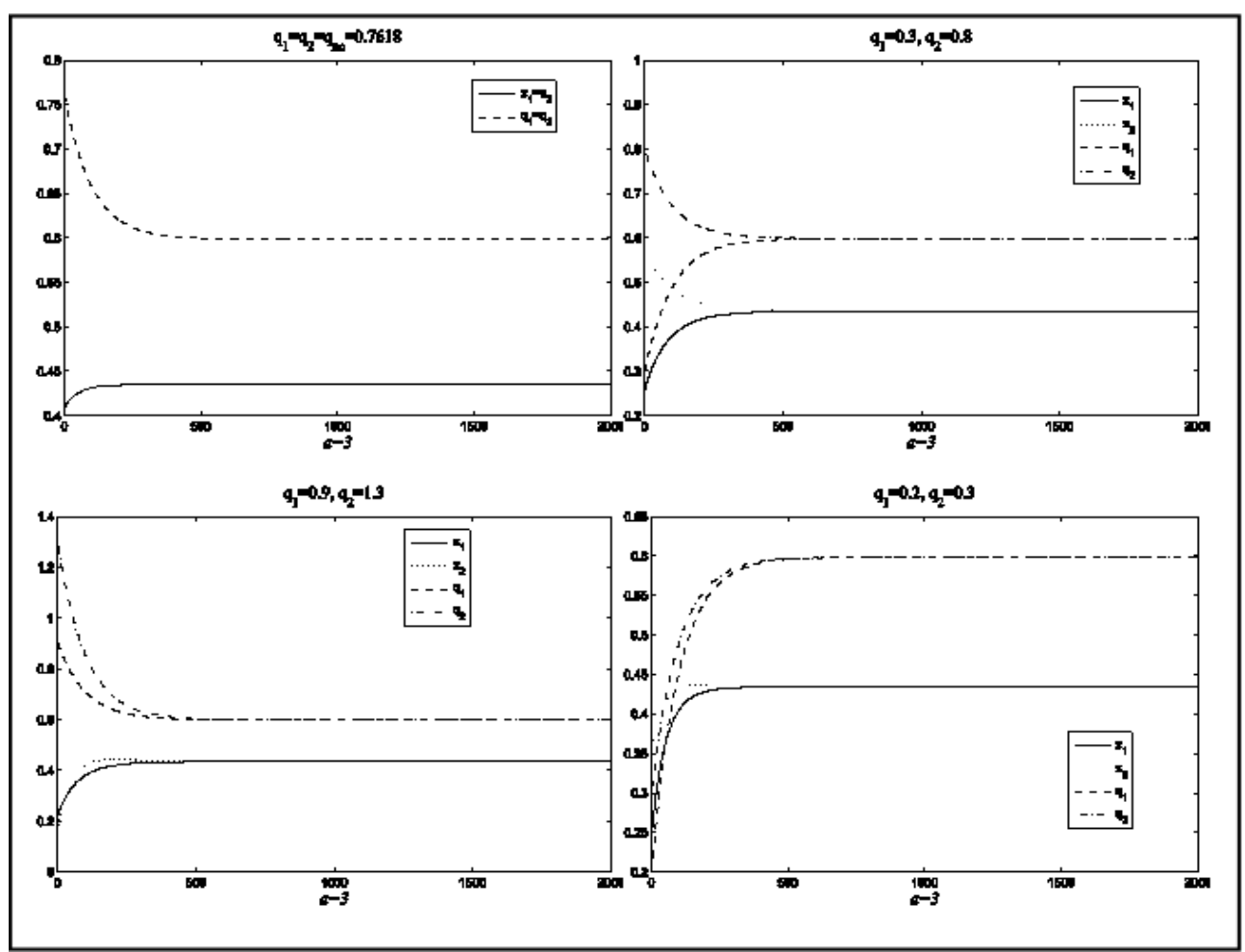


Como en 4.1 hemos manejado diferentes parámetros de ajuste. Hemos repetido las simulaciones con las mismas condiciones iniciales y $\alpha_{i}=0.01, \beta_{i}$ $=0.005$ y $\alpha_{i}=0.01, \beta_{i}=0.02$. Hemos de señalar que en este caso las diferencias son aún menores que en el modelo lineal tanto para $a=3$ como para $a=8$.

Figura 6.

Convergencia de beneficios y cantidades a los valores cooperativos para $a=8$. Las condiciones iniciales de cada simulación aparecen en el título de cada gráfica.

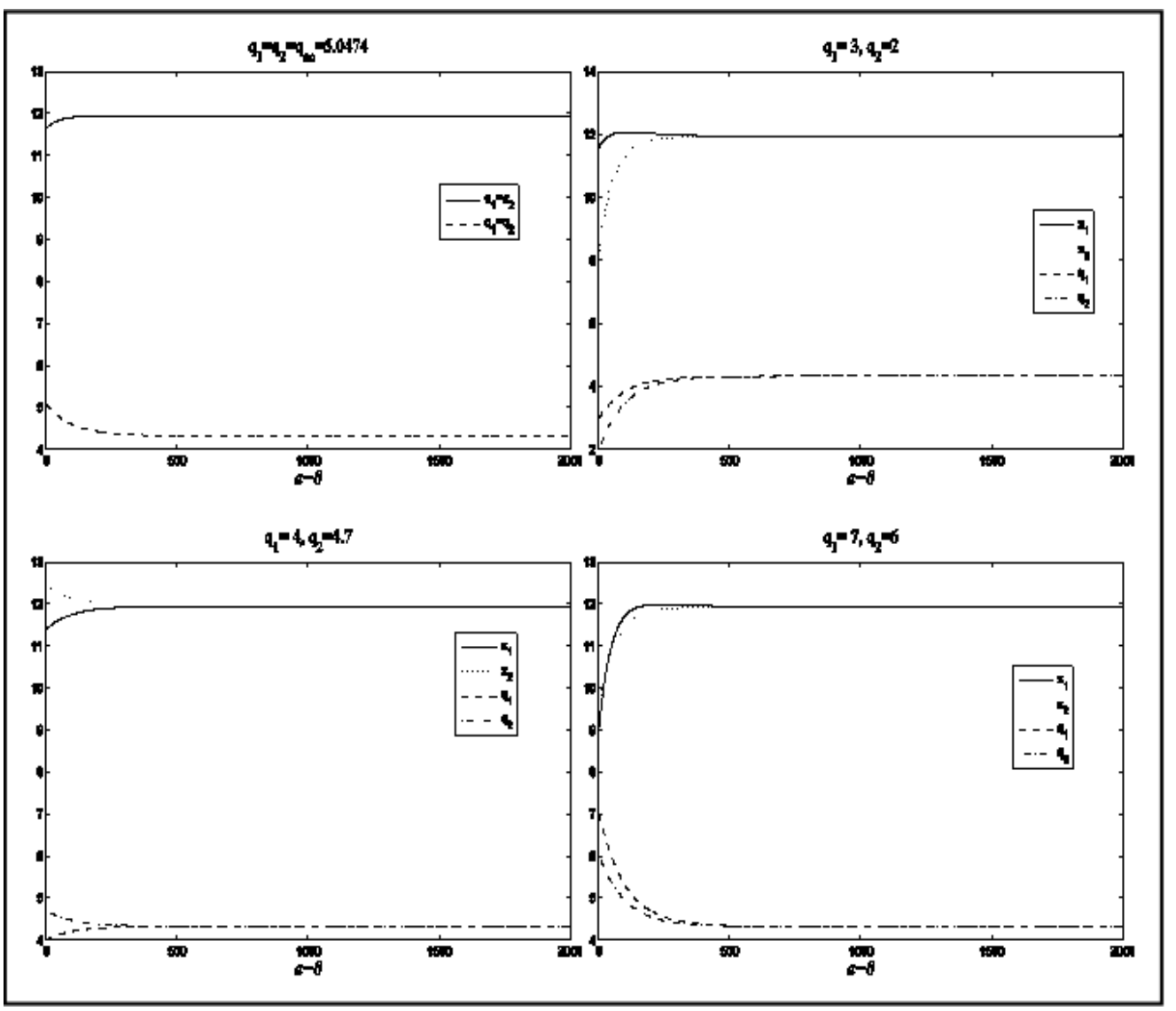

\subsection{Duopolio lineal con costes distintos}

En 4.1 y 4.2 hemos estudiado modelos donde los costes para las empresas son iguales. En este apartado nos planteamos el estudio de un modelo lineal asimétrico donde las empresas tienen diferente tamaño y los costes de producción son $c_{1}$ y $c_{2}$. Análogamente al caso en que los costes son iguales 
suponemos que $a>\max \left\{c_{1}, c_{2}\right\}$. En este modelo asimétrico, las cantidades en el equilibrio de Nash son

$$
q_{n c}^{1}=\frac{a-2 c_{1}+c_{2}}{3 b} \text { y } q_{n c}^{2}=\frac{a-2 c_{2}+c_{1}}{3 b} .
$$

Más comprometido es determinar en esta situación la solución cooperativa. Dado que las cantidades de equilibrio no son iguales, puede ser razonable que esta diferencia se conserve en las cantidades cooperativas. Podría argumentarse una reducción en un tanto por ciento de la cantidad de equilibrio como ocurre en el modelo lineal (el output cooperativo es el $75 \%$ del de Cournot), sin embargo, ello nos llevaría a no respetar la geometría del problema porque nos movemos sobre puntos de una recta que no pasa por los dos puntos de corte de las curvas de isobeneficio que determinan el equilibrio de Cournot (ver figura 7). Por ello, nos planteamos determinar las cantidades cooperativas de la misma forma empleada en el caso no lineal.

Figura 7.

Duopolio con costes distintos.

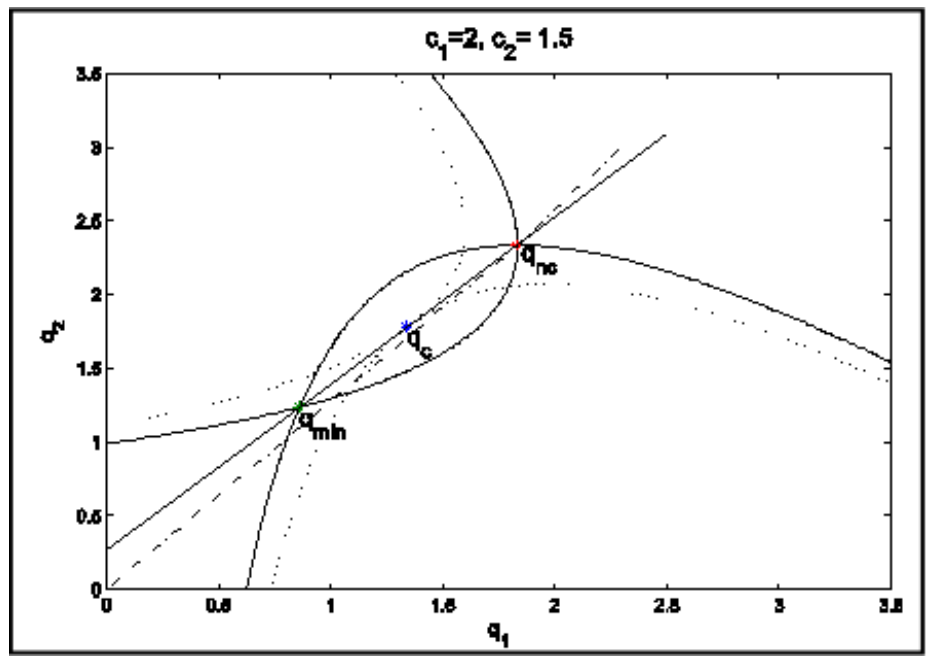

Vamos a estudiar la evolución de los beneficios para ambas empresas con producciones entre $q_{\min }=\left(q_{\min }^{1}, q_{\min }^{2}\right)$ y $q_{n c}=\left(q_{n c}^{1}, q_{n c}^{2}\right)$; es decir aquellas $\left(q_{1}\right.$, $q_{2}$ ) que satisfacen la restricción

$$
q_{2}=q_{n c}^{2}+\frac{q_{\min }^{2}-q_{n c}^{2}}{q_{\min }^{1}-q_{n c}^{1}}\left(q_{1}-q_{n c}^{1}\right) .
$$


En el caso simétrico, ambas empresas tienen la misma función de beneficios por lo que la determinación de la cantidad cooperativa no presenta problemas. En el modelo asimétrico, en principio podría ocurrir que obtuviésemos soluciones diferentes para cada una de las empresas lo que implicaría una dificultad añadida a la hora de establecer la solución cooperativa. Sin embargo esto no ocurre, el máximo de los beneficios para ambas empresas se alcanza para los mismos valores de las producciones.

El modelo concreto estudiado es el que tiene por parámetros $a=8, b=1, c_{1}$ $=2$ y $c_{2}=1.5$. En este caso los valores mínimos son $q_{\min }=(0.8615,1.2369)$. Los beneficios de ambas empresas son máximos en el punto $(1.3474,1.7851)$ que va a ser considerado como $q_{c}$. En la figura 8 se representan algunas de las simulaciones realizadas. Se han tomado las variaciones de los parámetros de ajuste como en los otros modelos. Como se puede apreciar la convergencia a las cantidades y beneficios cooperativos sigue las pautas de los casos simétricos. La diferencia es que ahora cada empresa tiene cantidades de referencia distintas.

\section{Figura 8}

Convergencia de beneficios y cantidades a los valores cooperativos para $a=8$. Las condiciones iniciales de cada simulación aparecen en el título de cada gráfica.

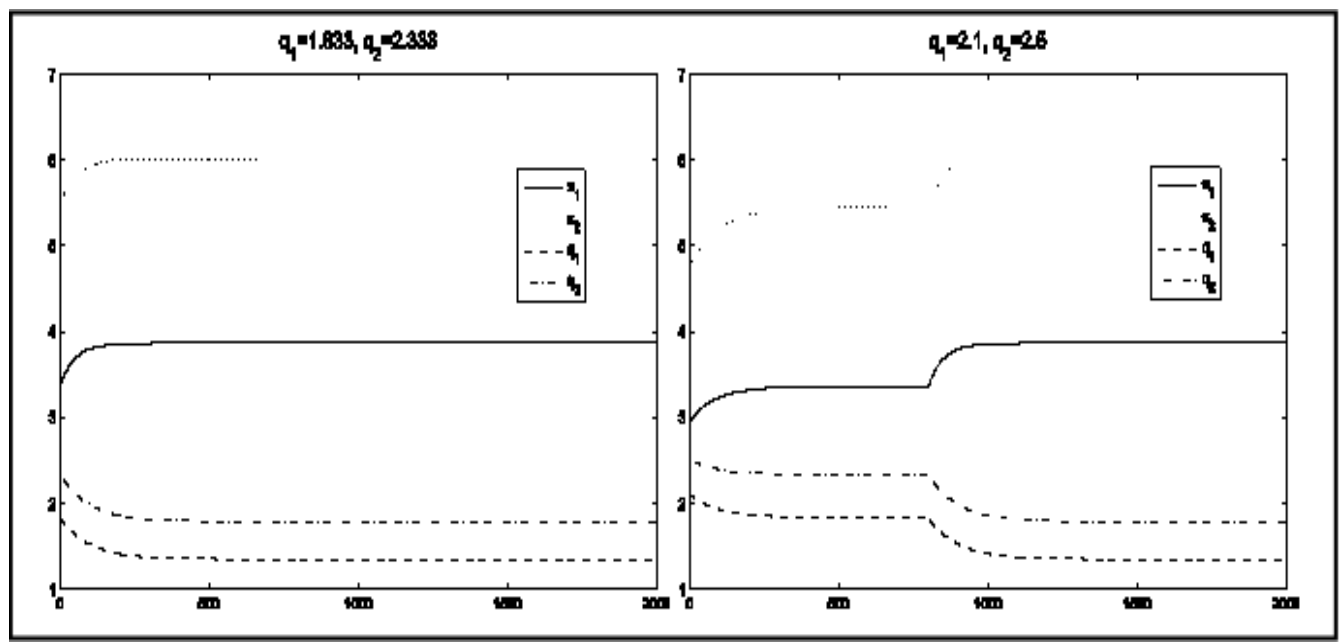

\section{CONCLUSIONES}

En este trabajo se estudia la emergencia de la cooperación en el oligopolio de Cournot cuando las empresas siguen un conjunto de reglas sencillas y objetivas en la determinación de sus producciones. El análisis muestra como la intención cooperativa es un aspecto fundamental para conseguir un incremento en los beneficios proporcionados por el equilibrio de Nash-Cournot. Si ambas 
empresas se proponen mejorar sus beneficios y siguen las estrategias expuestas en el trabajo, la conclusión es que lo consiguen. El modelo se desarrolla con información incompleta porque las empresas deciden sus producciones teniendo en cuenta sólo la información relativa a su propia empresa. Es cierto que en todos los modelos estudiados ambas empresas conocen los costes de producción de las otras aunque no las cantidades producidas. La extensión del modelo a un ámbito de mayor desinformación y sin duda más realista, es el paso siguiente dado que los resultados han sido realmente alentadores.

\section{REFERENCIAS BIBLIOGRÁFICAS}

AGIZA, H.N., ELSADANY, A.A. (2003). "Nonlinear dynamics in the Cournot oligopoly games with heterogeneous players" en Physica A, 320, 512-24.

AXELROD, R. (1984). "The evolution of cooperation". New York: Basic Books.

BISCHI, G.I., NAIMZADA, A.K., SBRAGIA, L. (2007). "Oligopoly games with local monopolistic approximation" en Journal of Economic Behavior and Organization, 62(3), 371-88.

CAFAGNA, V., COCCORESE, P. (2005). "Dynamical systems and the arising of cooperation in a Cournot duopoly" en Chaos, Solitons \& Fractals, 25, 65564.

DING, Z., SHI, G. (2009). "Cooperation in a dynamical adjustment of duopoly game with incomplete information" en Chaos, Solitons \& Fractals, 42, 98993.

FALK, A., FEHR, E., FISCHBACHER, U. (2008). "Testing theories of fairness-Intentions matter" en Games and Economic Behavior, 62, 287-03.

NAIMZADA AHMAD, K., SBRAGIA, L. (2006). "Oligopoly games with nonlinear demand and cost functions: two boundedly rational adjustment processes" en Chaos, Solitons \& Fractals, 29, 707-22.

NEGISHI, T. (1961). "Monopolistic competition and general equilibrium" en Review of Economic Studies, 27, 136-39.

PÉREZ, J., JIMENO, J.L. Y CERDÁ, E. (2004). Teoría de juegos, Madrid: Pearson Education, S.A.

SMALE, S. (1980). "The prisoner's dilemma and dynamical systems associated to non-cooperative games" en Econometrica, 48,1617-34.

SZIDAROVSZKY, F., OKUGUCHI, K. (1998). "An oligopoly model of commercial fishing" en Seoul Journal of Economics, 11, 417-42. 